\title{
Uso de anfotericina B deoxicolato y sus reacciones adversas en un hospital universitario en Chile
}

\author{
Roxanna Quinteros A., Alberto Fica C., Nancy Abusada A., \\ Lorena Muñoz C., Catalina Novoa M. y Carlos Gallardo A.
}

\section{Amphotericin B deoxycholate prescription and adverse events in a Chilean university hospital}

Amphotericin B deoxycholate is associated with infusion-related toxicity and renal toxicity. Purpose: To evaluate medical indications of this compound in a tertiary care center, analyze adverse reactions, infusion protocols and outcome of treated patients. Patients and methods: Retrospective analysis of 39 treatments indicated in 33 patients during 2007, exploring indications, infusion protocols and renal protective measures, infusion-related adverse reactions, nephrotoxicity, hypokalemia and outcomes. Results: On average, therapy lasted 12 days (2 to 39 ) and reached $600 \mathrm{mg}$ of accumulated dose (100 to 1950) respectively. 24-hours infusions were applied in $63.2 \%$ of prescriptions and $35.9 \%$ received a $4-6$ hour infusion schedule. In addition, $36.8 \%$ received daily a saline infusion before amphotericin. Adverse reactions were observed in $40 \%$ of treatments, predominating fever (25\%). Nonetheless, nephrotoxicity was infrequent $(9.4 \%)$, of low magnitude, only affecting patients without previous renal disease, and not requiring dialysis. Hypokalemia developed in $21.6 \%$ of treatments. More than half of medical indications were empirical (59\%), for presumed infections either by filamentous fungi or yeasts. In the subgroup with microbiological information, main indications were invasive aspergillosis ( $15.4 \%$ of total), systemic candidiasis $(12.8 \%)$ or meningeal cryptococcosis (10.3\%). A favorable response was registered in $41 \%$, and only $48.5 \%$ of patients survived. In a multivariate analysis, only age $>60$ years remained as an independent factor for developing infusion-related adverse reactions. In the same manner, a SOFA score $>3$ and corticosteroids administration at the same time than amphotericin B, were independently associated to a fatal outcome. Conclusion: infusion-related adverse reactions are frequent during amphotericin B deoxycholate therapy, but renal toxicity is occasionally observed. Amphotercin B was used mainly as empirical therapy in this study.

Key words: Amphotericin B deoxycholate, drug toxicity, nephrotoxicity, utilization.

Palabras clave: Anfotericina B deoxicolato, efectos adversos, utilización, nefrotoxicidad.

\section{Introducción}

$\mathrm{L}$ as infecciones fúngicas sistémicas se han convertido en un problema en incremento en diversos tipos de pacientes incluyendo aquellos con neutropenia febril, trasplante de precursores hematopoyéticos (TPH) u órganos sólidos (TOS), usuarios de corticoesteroides a dosis altas y también en pacientes sometidos a cirugía abdominal o portadores de catéteres vasculares centrales $^{1-4}$. Los agentes involucrados, dependiendo del tipo de paciente, incluyen hongos filamentosos, fundamentalmente del género Aspergillus y levaduras del género Candida ${ }^{1-4}$.

Una infección fúngica sistémica en pacientes de este tipo es grave, por lo que requiere un tratamiento oportuno y eficaz para combatirla. En las últimas tres décadas, el agente de elección para iniciar el tratamiento de la mayoría de las micosis sistémicas ha sido la anfotericina
B deoxicolato 5 , la que a pesar de los efectos tóxicos descritos en la literatura mundial, continúa teniendo un papel importante en el tratamiento de las infecciones micóticas sistémicas ${ }^{6}$. Sólo en la última década su sitial se ha visto en parte destronado por el advenimiento de moléculas o preparados más seguros y ocasionalmente de mayor eficacia ${ }^{7-9}$. No obstante, anfotericina B deoxicolato, sigue siendo un fármaco socorrido por su amplio espectro hacia levaduras y hongos filamentosos, perfil de toxicidad conocido, el que es posible de ser monitorizado y un costo relativamente bajo. Las presentaciones lipídicas son muy onerosas a pesar de su mejor perfil de seguridad lo que explica su uso ocasional en Chile.

La administración de anfotericina B deoxicolato se puede asociar a efectos adversos inmediatos, tales como fiebre, calofríos, náuseas, vómitos, cefalea, shock anafiláctico, arritmias y falla hepática, pero también reacciones adversas que se presentan después de administraciones
Hospital Clínico Universidad de Chile, Santiago Servicio de Farmacia (RQA) Departamento de Medicina (CNM, CGA) Unidad de Abastecimiento (LMC) Hospital Militar de Santiago, Chile Servicio de Infectología (AFC)

Recibido: 11 de abril de 2009 Aceptado: 27 de octubre de 2009

Correspondencia a: Alberto Fica Cubillos albertofica@gmail.com 
repetidas del medicamento, incluyendo en estas últimas hipokalemia, hipomagnesemia y toxicidad medular. Sin embargo, la toxicidad más preocupante y en la cual se ha centrado la mayoría de los estudios sigue siendo la nefrotoxicidad 9 .

Escasa información está disponible en Chile sobre la toxicidad de este compuesto y sólo se dispone de cuatro publicaciones sobre este tópico ${ }^{9-12}$, dos de ellas de revisión, una sobre eficacia y efectos adversos en una serie de 10 pacientes y la última sobre toxicidad comparativa entre anfotericina deoxicolato (convencional) y formulaciones lipídicas.

Sobre esta base, se propuso averiguar por qué se indica este compuesto, cómo se utiliza, qué reacciones adversas se observan, el desenlace de los pacientes tratados y los factores potencialmente involucrados.

\section{Materiales y Métodos}

Se efectuó un estudio retrospectivo con el universo de tratamientos con anfotericina B deoxicolato efectuados durante el año 2007. La unidad de análisis fue la ficha clínica y la información de los pacientes fue recopilada desde el sistema de farmacia y los registros de vigilancia del programa de uso de anfotericina del Hospital Clínico de la Universidad de Chile, utilizando para ello una hoja estandarizada de recolección de información.

Criterios de inclusión. Se incluyeron todos aquellos pacientes que estuvieron en tratamiento con anfotericina $\mathrm{B}$ deoxicolato por diagnóstico microbiológico o con sospecha clínica de micosis sistémica y que al menos recibieron $100 \mathrm{mg}$ de este fármaco.

Variable clínicas y de laboratorio. Se registraron datos bio-demográficos de los pacientes, fecha de ingreso y egreso, traslado desde o hacia otros centros o re-ingreso al hospital, antecedentes mórbidos, característica de la terapia como medicamentos concomitantes nefrotóxicos, dosis diaria y total de anfotericina $\mathrm{B}$, indicación para su uso ya fuese como sospecha de infección fúngica, infección fúngica confirmada o por profilaxis. También se consignó el valor SOFA (Sepsis-related Organ Failure Assessment) el día de comienzo de la terapia ${ }^{13}$.

Evaluación de la toxicidad. La toxicidad se evaluó en forma independiente respecto a la infusión como al tratamiento prolongado.

Toxicidad asociada a infusión. Se consideraron como tal, fiebre, escalofríos, vómitos y/o náuseas. Se definió aparición de fiebre relacionada a la infusión si hubo al menos una elevación de la $\mathrm{t}^{\mathrm{o}}$ de $1^{\circ} \mathrm{C}$ sobre la línea basal, al inicio, durante o al completar la infusión ${ }^{14}$. Los calofríos y los vómitos y náuseas fueron pesquisados desde la ficha clínica y asignados como efecto asociado a la infusión si ocurrieron durante la administración de ella.

Nefrotoxicidad e hipokalemia. Se utilizaron los mismos criterios considerados por otros autores para definir nefrotoxicidad ${ }^{15}$. Para aquellos pacientes con función renal previa normal, se definió nefrotoxicidad como el incremento de la creatininemia a un valor $\geq 2$ $\mathrm{mg} / \mathrm{dL}$. Para pacientes con función renal previa anormal, se consideró como nefrotoxicidad, el aumento al doble de la creatininemia inicial ${ }^{15}$. Se registró también, el uso concomitante de fármacos nefrotóxicos tales como vancomicina, amikacina o ciclosporina. También se evaluó el desarrollo de hipokalemia asociada al tratamiento y que fue definida como una kalemia $<3,5 \mathrm{mEq} / \mathrm{L}$ durante el uso de anfotericina $\mathrm{B}^{14}$.

Casos de aspergilosis. Los casos de aspergilosis invasora fueron catalogados como probados, probables o posibles de acuerdo a criterios estandarizados ${ }^{16}$.

Evaluación de la respuesta clínica. La respuesta al tratamiento fue catalogada de favorable si se observó una mejoría clínica y de fracaso si el paciente falleció durante el tratamiento o permaneció en condiciones clínicas estacionarias. Aquellos casos en los cuales no hubo seguimiento del paciente hasta el término del tratamiento con anfotericina $\mathrm{B}$ fueron asignados como no evaluables.

Análisis estadístico. Se utilizó la prueba bilateral de Fisher para variables categóricas y el test de Wilcoxon para muestras pareadas continuas con un nivel de significación de 0,05 . Cuando se identificaron varios factores de riesgo se procedió a efectuar un análisis multivariado de regresión logística binaria usando el método de entrada. Los cálculos fueron apoyados con el programa SPSS v 14.0.

\section{Resultados}

Características generales de la población. Un total de 39 tratamientos en 33 pacientes fue identificado en los 12 meses del período bajo análisis. Treinta pacientes recibieron un solo curso, uno recibió dos esquemas, otro, tres tratamientos y el último caso cuatro cursos terapéuticos. La edad promedio de los pacientes fue de 45 años (rango de 18 a 76 años) con predominio del sexo masculino ( $\mathrm{n}=22 ; 66,7 \%$ ).

Entre los pacientes destacaba el antecedente de cáncer hematológico (36,4\%), insuficiencia renal crónica (21\%) o infección por VIH/SIDA (15,2\%, Tabla 1$)$. Una fracción menor tenía antecedentes de TPH, diabetes mellitus o TOS. Además, sólo una baja proporción de los pacientes tenía cáncer sólido no linfoma (Tabla 1). 
Durante el tratamiento, cerca de la mitad de los pacientes tenía antecedentes de uso previo de corticosteroides en dosis inmunosupresoras $(53,8 \%$, Tabla 1$)$, cerca de un tercio neutropenia $<500 / \mathrm{mm}^{3}, 23 \%$ diálisis y $17 \%$ insuficiencia hepática. El antecedente de cirugía abdominal electiva o de urgencia fue infrecuente (Tabla 1).

Atendiendo a los antecedentes mórbidos de los pacientes, $28 \%$ estuvo o estaba hospitalizado en UCI cuando se planteó el tratamiento con este compuesto y un tercio en unidades onco-hematológicas (Tabla 1). Además, cerca de $40 \%$ de los eventos estuvieron ligados a re-ingresos o traslados desde otros centros asistenciales.

Infecciones tratadas con anfotericina $B$. Este fármaco fue utilizado por diferentes razones, siendo la más frecuente el uso empírico en sospecha de aspergilosis pulmonar invasora $(33,3 \%$, Tabla 2$)$. El tratamiento de aspergilosis pulmonar invasora, documentada por biopsia, antigenemia o con infiltrados sugerentes radiológicos, $(15,4 \%)$ y el tratamiento por sospecha $(2,6 \%)$ o en forma confirmada de mucormicosis $(2,6 \%)$, completan las otras indicaciones iniciadas por hongos filamentosos, las que en conjunto engloban poco más de las mitad de los tratamientos (Tabla 2).

Un tercio de los tratamientos fue iniciado para el manejo o sospecha de infecciones por levaduras, ya fuese del género Candida o Cryptococcus neoformans. Dentro de este grupo, las indicaciones más frecuentes fueron la sospecha de candidiasis sistémica $(10,3 \%)$ y el tratamiento de pacientes con meningitis criptococóccica $(10,3 \%)$. También se detectaron casos con indicaciones por candidemia $(\mathrm{n}=1 ; 2,6 \%)$, fracaso de triazoles en caso de candidiasis sistémica $(5,1 \%)$, contraindicación para su uso $(2,6 \%)$ o resistencia del agente causal a estos compuestos $(2,6 \%$, Tabla 2$)$.

En cuatro casos adicionales se utilizó anfotericina B como profilaxis, ya fuese de aspergilosis pulmonar en pacientes neutropénicos $(n=3)$ o para prevenir candidiasis sistémica en un paciente con múltiples cirugías abdominales y con una larga estadía en UCI, no utilizándose fluconazol ( $n=1$, Tabla 2). En otro caso se utilizó anfotericina B para cubrir simultáneamente la sospecha de candidiasis y aspergilosis en un paciente con antecedentes de trasplante hepático (Tabla 2).

Características del tratamiento. La duración promedio del tratamiento fue de 12 días (rango de 2 a 39 días). La dosis promedio utilizada en los pacientes fue de $50 \mathrm{mg} /$ día (rango 25 - $80 \mathrm{mg}$ ). La dosis acumulativa de anfotericina $\mathrm{B}$ administrada fue en promedio $600 \mathrm{mg}$ (rango 100-1.950 $\mathrm{mg}$ ). El tipo de infusión para el tratamiento de anfotericina B estuvo distribuida en infusión de 24 horas $(63,2 \%)$ o en 4 a 6 horas $(35,9 \%)$. Además, en $54,8 \%$ de los pacientes se pudo objetivar el uso de otras medidas profilácticas para disminuir efectos adversos, administrando ya sea una precarga salina antes de la infusión del medicamento (62,8 $\%$ ) o corticosteroides sistémicos o en el matraz junto con la infusión de anfotericina B (38,1\%) (Tabla 3).

En $62,8 \%$ de los pacientes se utilizó otro antifúngico antes de iniciar el tratamiento con anfotericina B (mayoritariamente fluconazol), y otro $42,9 \%$ recibió otro antifúngico después del tratamiento con anfotericina B. En una minoría (13,5\%), los pacientes recibieron un tratamiento combinado con otro antifúngico y que correspondió en todos los casos a caspofungina $(n=5 ; 100 \%)$. (Tabla 3$)$.

Datos microbiológicos. No todos los casos contaban con información microbiológica sobre la especie de hongos que se asoció a la infección para poder justificar el uso de anfotericina B y sólo en 16 tratamientos se pudo identificar el agente potencial: Cinco casos presentaron infección por Candida spp (12,8\%), cinco cuadros posi-

Tabla 1. Características generales de los 33 pacientes y de los 39 tratamientos que recibieron anfotericina $B$ deoxicolato

\begin{tabular}{|c|c|c|}
\hline Condición & $\begin{array}{l}\text { Frecuencia o valor } \\
\text { promedio }\end{array}$ & $\begin{array}{l}\text { Porcentaje } \\
\text { (o rango) }\end{array}$ \\
\hline $\operatorname{Sexo}(M / F)$ & $22 / 11$ & $66,7 / 33,3 \%$ \\
\hline Edad (años) & 45 & $18-76$ \\
\hline Cáncer hematológico & 12 & $36,4 \%$ \\
\hline IRC & 7 & $21,2 \%$ \\
\hline Trasplante de precursores hematopoyéticos & 4 & $12,1 \%$ \\
\hline Infección por VIH / SIDA & 5 & $15,2 \%$ \\
\hline Diabetes mellitus & 3 & $9,1 \%$ \\
\hline Trasplante de órgano sólido & 3 & $9,1 \%$ \\
\hline EPOC & 3 & $9,1 \%$ \\
\hline Cáncer sólido no linfoma & 1 & $3,0 \%$ \\
\hline \multicolumn{3}{|l|}{ Durante el tratamiento } \\
\hline Usuario de corticosteroides & 21 & $53,8 \%$ \\
\hline Neutropenia $<500 / \mathrm{mm}^{3}$ & 12 & $30,8 \%$ \\
\hline Diálisis & 9 & $23,1 \%$ \\
\hline Insuficiencia hepática & 7 & $17,9 \%$ \\
\hline Cirugía abdominal electiva & 3 & $7,7 \%$ \\
\hline Cirugía abdominal de urgencia & 2 & $5,1 \%$ \\
\hline \multicolumn{3}{|l|}{$\begin{array}{l}\text { Unidades de hospitalización y traslados } \\
\text { antes del tratamiento }\end{array}$} \\
\hline Estadía en Unidad de Cuidados intensivos & 11 & $28,2 \%$ \\
\hline Estadía en unidad onco-hematológica & 13 & $33,3 \%$ \\
\hline Estadía en unidad nefrológica & 3 & $7,7 \%$ \\
\hline Re-ingreso o traslado & 16 & $41,0 \%$ \\
\hline
\end{tabular}


Tabla 2. Indicaciones de uso en 39 terapias con anfotericina B

\begin{tabular}{|c|c|c|}
\hline Indicación de uso de anfotericina B & $\begin{array}{l}\text { Frecuencia } \\
(\mathrm{n}=44)\end{array}$ & $\%$ \\
\hline $\begin{array}{l}\text { Uso empírico por sospecha de aspergilosis } \\
\text { pulmonar invasora }\end{array}$ & 13 & $33,3 \%$ \\
\hline $\begin{array}{l}\text { Tratamiento de aspergilosis pulmonar invasora o } \\
\text { nasal probada, probable o posible }\end{array}$ & 6 & $15,4 \%$ \\
\hline Sospecha de mucormicosis & 1 & $2,6 \%$ \\
\hline Tratamiento de mucormicosis & 1 & $2,6 \%$ \\
\hline Subtotal hongos filamentosos & 21 & $53,8 \%$ \\
\hline $\begin{array}{l}\text { Uso empírico por sospecha de candidiasis } \\
\text { sistémica }\end{array}$ & 4 & $10,3 \%$ \\
\hline $\begin{array}{l}\text { Tratamiento de candidiasis sistémica en pacientes } \\
\text { con fracaso de triazoles }\end{array}$ & 2 & $5,1 \%$ \\
\hline $\begin{array}{l}\text { Tratamiento de candidiasis sistémica en pacientes } \\
\text { con contraindicación de uso de triazoles }\end{array}$ & 1 & $2,6 \%$ \\
\hline $\begin{array}{l}\text { Tratamiento de meningitis por Cryptococcus } \\
\text { neoformans }\end{array}$ & 4 & $10,3 \%$ \\
\hline $\begin{array}{l}\text { Tratamiento de candidadiasis resistente a } \\
\text { triazoles }\end{array}$ & 1 & $2,6 \%$ \\
\hline Tratamiento de candidemia & 1 & $2,6 \%$ \\
\hline Subtotal levaduras & 13 & $33,3 \%$ \\
\hline \multicolumn{3}{|l|}{ Otras condiciones } \\
\hline Profilaxis de infecciones fúngicas & 4 & $10,3 \%$ \\
\hline Otras & 1 & $2,6 \%$ \\
\hline Total & 39 & $100,0 \%$ \\
\hline
\end{tabular}

\begin{tabular}{|c|c|c|}
\hline Características del tratamiento & \multicolumn{2}{|c|}{ Valor promedio o frecuencia } \\
\hline Duración de la terapia días (rango) & 12 & $(2-39)$ \\
\hline Dosis diaria mg (rango) & 50 & $(25-80)$ \\
\hline Dosis acumulada mg (rango) & 600 & $(100-1.950)$ \\
\hline Otro antifúngico antes del tratamiento & 25 & $(64,1 \%)$ \\
\hline Otro antifúngico durante el tratamiento & 5 & $(12,8 \%)$ \\
\hline Otro antifúngico después del tratamiento & 17 & $(43,6 \%)$ \\
\hline Otros fármacos nefrotóxicos & 28 & $(71,8 \%)$ \\
\hline Infusión por 24 hs (sobre 38 tratamientos) & 24 & $(63,2 \%)$ \\
\hline Infusión de 4 a 6 hs (sobre 38 tratamientos) & 14 & $(36,8 \%)$ \\
\hline Precarga salina (sobre 38 tratamientos) & 20 & $(52,6 \%)$ \\
\hline Corticoides sistémicos & 22 & $(56,4 \%)$ \\
\hline Corticoides en matraz & 14 & $(35,9 \%)$ \\
\hline
\end{tabular}

bles y uno probado por Aspergillus sp $(15,4 \%)$, cuatro por $C$. neoformans $(10,3 \%)$ y un caso de mucormicosis $(2,6 \%)$. En los demás casos no fue posible identificar el agente potencial que produjo la infección (59\%) (Tabla 4).

Reacciones adversas. Durante la infusión del fármaco se presentaron reacciones adversas en 18 cursos de terapia, siendo fiebre la más frecuente ( $25 \%)$; vómitos se presentaron en $15,2 \%(5 / 33)$, calofríos en $7,1 \%(2 / 28)$ y flebitis en $6,7 \%$ (Tabla 5). Al menos $40 \%$ de los tratamientos se asoció a alguna de estas cuatro manifestaciones clínicas. La probabilidad de presentar alguna de estas manifestaciones aumentó significativamente cuando el paciente tenía un valor SOFA $>3$, era mayor de 60 años o fue tratado por aspergilosis (Tabla 6). En el análisis multivariado, sólo la edad > 60 años permaneció como factor independiente para el desarrollo de estos problemas (Tabla 6). La no utilización de precarga salina o el uso de infusiones de 4 a 6 horas no se asociaron a un mayor riesgo, al igual que el tipo de patología del paciente, el uso de otros antifúngicos, la dosis acumulada de anfotericina $\mathrm{B}$ o la prescripción de estimuladores de colonias. Tampoco este riesgo disminuyó con el uso de corticosteroides ya fuese en el matraz o en forma sistémica (datos no mostrados).

Se desarrolló nefrotoxicidad en tres de 32 tratamientos $(9,4 \%)$ en los cuales se contó con información de seguimiento de la función renal. La nefrotoxicidad sólo fue observada entre pacientes con función renal previa normal y ningún paciente requirió diálisis por causa directa del fármaco (Tablas 7 y 8). La hipokalemia (potasemia $<3,5 \mathrm{mEq} / \mathrm{L})$ se presentó en ocho tratamientos $(21,6 \%)$ (Tabla 7). Para el subgrupo de pacientes sin antecedentes de falla renal previo al ingreso, el valor promedio de la creatininemia al inicio del tratamiento con anfotericina B fue de $0,86 \mathrm{mg} / \mathrm{dL} \pm 0,40$, de $1,30 \mathrm{mg} / \mathrm{dL} \pm 0,54$ en la mitad del tratamiento y de $1,18 \mathrm{mg} / \mathrm{dL} \pm 0,50$ al finalizar el tratamiento (Tabla 8). Aunque las diferencias entre los valores a la mitad o al final de tratamiento respecto al valor inicial fueron significativas por una prueba no paramétrica, su magnitud fue discreta. En el subgrupo de pacientes con falla renal conocida, las diferencias observadas en diferentes momentos del seguimiento no fueron significativas (Tabla 8).

Respuesta al tratamiento. Al considerar el conjunto global de tratamientos, $46,2 \%$ tuvo una respuesta no favorable ya fuese porque el paciente falleció durante el tratamiento, tuvo una situación estacionaria o un deterioro en su condición clínica. Sólo tres de los 18 pacientes con una respuesta no favorable recibieron otro compuesto antifúngico (16,7\%) luego de la anfotericina B ya que los 15 restantes fallecieron. Un $41 \%$ de los pacientes presentó una respuesta favorable al tratamiento. Finalmente, en $12,8 \%$ de los tratamientos, la evolución no fue evaluable 
por traslado del paciente o interrupción precoz, por no confirmarse el diagnóstico clínico con cultivos (Tabla 9).

Al analizar el subgrupo con terapia dirigida microbiológicamente, se observó una respuesta favorable para levaduras en 33,3\% $(\mathrm{n}=3)$, no favorable en $55,6 \%(\mathrm{n}=$ $5)$ y no evaluable en $11,1 \%$ restante $(n=1)$. En el caso de infecciones por hongos filamentosos se pesquisó $42,9 \%$ de respuestas favorables $(n=3)$ y $57,1 \%$ de respuestas no favorables $(n=4)$. Las diferencias en las respuestas observadas para levaduras y hongos filamentosos no fueron significativas.

Condición al egreso. Cerca de la mitad de los pacientes egresaron vivos al terminar su tratamiento $(\mathrm{n}=22$; $56,4 \%$ ). Un total de 17 pacientes falleció representando $43,6 \%$ de los 39 egresos y $51,5 \%$ de los 33 pacientes.

\section{Análisis de factores asociados a nefrotoxicidad y respuesta clínica}

Se analizaron diferentes factores asociados al riesgo de presentar nefrotoxicidad tales como edad, dosis acumulada, duración del tratamiento, uso concomitante de otros fármacos nefrotóxicos, shock al inicio del tratamiento, uso de corticosteroides en forma paralela, infusiones de 4 a 6 horas, no uso de precarga salina o el haber recibido cursos previos con anfotericina $\mathrm{B}$ y ninguno de ellos resultó asociado a un mayor riesgo de nefrotoxicidad (datos no mostrados).

En la respuesta clínica se analizaron varios factores que se pudieran relacionar con una evolución, encontrando ocho factores que se asociaron a un desenlace fatal: antecedentes de IRC en hemodiálisis, hemodiálisis o shock al inicio del tratamiento, la asociación de otro antifúngico con anfotericina $\mathrm{B}$, antecedentes de uso de corticosteroides al ingreso o en forma paralela al tratamiento, valor SOFA $>3$ y aparición de fiebre durante la terapia (Tabla 10). Varios de estos factores de riesgo o protectores representan condiciones sobrepuestas y por ello se procedió a efectuar un análisis multivariado. En este último proceso sólo el valor SOFA y el uso concomitante de corticosteroides en forma sistémica permanecieron significativos (Tabla 10).

\section{Discusión}

Existe escasa información publicada sobre tratamientos con anfotericina B y su toxicidad en Chile. Hernández y cols, investigaron en una población de pacientes críticos con candidiasis sistémica demostrada o probable las reacciones adversas que se produjeron durante el tratamiento con anfotericina B convencional o anfotericina B en emulsión lipídica artesanal $\left(\right.$ Intralipid $^{\circledR}$ ). Fue un estudio de cohorte donde 17 pacientes recibieron anfotericina $B$ convencional y 28 la emulsión lipídica. En esa serie no

\begin{tabular}{|lcc|}
\multicolumn{4}{|c|}{ Tabla 4. Tratamiento dirigido por datos microbiológicos } \\
\hline Agente & Frecuencia & $\%$ \\
\hline Candida sp & 5 & 12,8 \\
Aspergillus sp* & 6 & 15,4 \\
\hline Mucormicosis & 1 & 2,6 \\
Cryptococcus sp & 4 & 10,3 \\
Subtotal con datos microbiológicos & 16 & 41,0 \\
Sin diagnóstico microbiológico & 23 & 59,0 \\
\hline Total & 39 & 100 \\
\hline *: 5 casos posibles y uno probado & & \\
\hline
\end{tabular}

\begin{tabular}{|lccc|}
\hline & $\begin{array}{l}\text { Tabla 5. Reacciones adversas relacionadas a la infusión durante } \\
\text { el tratamiento con anfotericina B deoxicolato }\end{array}$ \\
\hline & $\begin{array}{c}\text { Tratamientos con } \\
\text { información disponibles }\end{array}$ & Frecuencia & $\%$ \\
\hline Fiebre & 36 & 9 & 25,0 \\
\hline Vómitos y/o náuseas & 33 & 5 & 15,2 \\
\hline Calofríos & 28 & 2 & 7,1 \\
Flebitis & 30 & 2 & 6,7 \\
\hline Cualquiera anterior & 30 & 12 & 40,0 \\
\hline
\end{tabular}

Tabla 6. Análisis de factores asociados al desarrollo de reacciones adversas durante la infusión de anfotericina B

$\begin{array}{ccc}\text { Factor } & \begin{array}{c}\text { Con } \\ \text { reacciones } \\ \text { adversas } \\ (n=12)\end{array} & \begin{array}{c}\text { Sin } \\ \text { reacciones } \\ \text { adversas } \\ (n=18)\end{array}\end{array}$

\section{Univariado}

$\begin{array}{lrllll}\text { SOFA }>3^{*} & 9 & 8 & 1,91 \quad(1,10-3,29) & <0,05 \\ \text { Tratamiento de aspergilosis** } & 5 & 0 & 3,57 \quad(1,90-6,69) & <0,01 \\ \text { Edad }>60 \text { años (si/no) } & 10 & 7 & 3,82(1,006-14,53) & <0,05\end{array}$

\section{Multivariado}

Edad $>60$ años

$15 \quad(1,03-218)<0,05$

SOFA: Sepsis-related Organ Failure Assessment. *: sobre 10 casos con reacciones adversas y 17 sin ellas. **: No incluye un caso de aspergilosis en el que no se contaba con todos los datos de reacciones adversas

Tabla 7. Nefrotoxicidad y/o hipokalemia durante el período del tratamiento con anfotericina B deoxicolato

\begin{tabular}{lccc} 
Condición & Frecuencia & $\begin{array}{c}\text { Población } \\
\text { con datos }\end{array}$ & $\%$ \\
\hline Nefrotoxicidad & 3 & 26 & 11,5 \\
\hline En pacientes con función renal previa normal & 0 & 6 & 0,0 \\
\hline En pacientes con función renal previa anormal & 3 & 32 & 9,4 \\
\hline Total nefrotoxicidad & 8 & 37 & 21,6 \\
\hline Hipokalemia & & &
\end{tabular}




\section{Tabla 8. Creatininemia al inicio y al final de la terapia con anfotericina B}

\section{deoxicolato}

\section{Creatininemia}

Número de tratamientos con información disponible

$\mathbf{m g} / \mathrm{dL} \pm \mathrm{SD}$

\section{En pacientes sin antecedentes de falla renal}

$\begin{array}{lll}\text { Valor inicial } & 28 & 0,86 \pm 0,40 \\ \text { Valor en la mitad del tratamiento } & 26 & 1,30 \pm 0,54^{*} \\ \text { Valor final } & 27 & 1,18 \pm 0,50^{* *}\end{array}$

En pacientes con antecedentes de falla renal

$\begin{array}{lll}\text { Valor inicial } & 6 & 2,38 \pm 1,39 \\ \text { Valor en la mitad del tratamiento } & 6 & 2,91 \pm 1,44 \\ \text { Valor final } & 5 & 2,12 \pm 1,11\end{array}$

*diferencias significativas por prueba de Wilcoxon $\mathrm{p}<0,0001$ (valor en la mitad respecto al inicial). **diferencias significativas por prueba de Wilcoxon $p<0,1$ (valor final respecto al inicial)

Tabla 9. Respuesta al tratamiento en 39 cursos de terapia empírica o dirigida con anfotericina B deoxicolato

\begin{tabular}{|lcc|}
\hline Respuesta al tratamiento & Frecuencia & (\%) \\
\hline Favorable & 16 & 41,0 \\
No favorable & 18 & 46,2 \\
No evaluable & 5 & 12,8 \\
Total & 39 & 100 \\
\hline
\end{tabular}

se pudo demostrar ventajas comparativas de anfotericina B en emulsión lipídica respecto a la formulación convencional $^{11}$. En otra serie con sólo 10 pacientes tratados en la década de los 80, Espinoza y cols, reportaron tres casos con reacciones adversas asociadas a la infusión, dos con deterioro de la función renal y nueve casos con hipokalemia. ${ }^{12}$ Finalmente, Cruz, en una revisión bibliográfica sobre el uso de anfotericina B intralípidica y convencional, determinó la diferencia en eficacia y nefrotoxicidad entre ellas; para esta búsqueda seleccionaron nueve estudios clínicos randomizados, doble ciego y un meta-análisis y se incluyó un trabajo chileno en cohorte (el trabajo antes mencionado). De 10 trabajos analizados en esta revisión, ocho mostraron menor nefrotoxicidad en los pacientes que fueron tratados con anfotericina B liposomal respecto a aquellos tratados con anfotericina $\mathrm{B}$ deoxicolato ${ }^{10}$. De esta manera, se puede concluir que la información que ha sido generada en Chile sobre la toxicidad de este medicamento es muy escasa.

Nuestro trabajo tuvo como propósito aportar más información sobre sus indicaciones, formas de uso, reacciones adversas asociadas al uso de este medicamento y el desenlace de los pacientes tratados. Para ello se diseñó un estudio retrospectivo que permitiera englobar una mayor cantidad de pacientes, con diversas patologías base, tanto críticos como de menor gravedad, con diferentes etiologías y de esta manera obtener un panorama representativo del escenario de uso de este compuesto y sus complicaciones. Nuestra serie reclutó 39 tratamientos en 33 pacientes a lo largo de un año de seguimiento.

\begin{tabular}{|c|c|c|c|c|}
\hline Factor & $\begin{array}{c}\text { Vivos } \\
(n=22)\end{array}$ & $\begin{array}{c}\text { Fallecidos } \\
(n=17)\end{array}$ & OR $\left(\mathrm{IC}_{95}\right)$ & $\mathbf{P}$ \\
\hline \multicolumn{5}{|l|}{ Univariado } \\
\hline IRC en hemodiálisis & 2 & 7 & $2,33(1,26-4,13)$ & $<0,05$ \\
\hline Hemodiálisis al inicio del tratamiento & 2 & 7 & $2,33(1,26-4,33)$ & $<0,05$ \\
\hline Shock al inicio del tratamiento & 2 & 7 & $2,57(1,38-4,81)$ & $<0,05$ \\
\hline SOFA $>3^{*}$ & 11 & 16 & $7,82(1,17-52,3)$ & $<0,01$ \\
\hline Terapia con otro antifúngico & 0 & 5 & $2,83(1,79-4,46)$ & $<0,05$ \\
\hline Usuario de corticosteroides al ingreso & 8 & 13 & $2,78(1,10-7,04)$ & $<0,05$ \\
\hline Corticosteroides sistémicos con anfotericina B & 8 & 14 & $3,60(1,23-10,55)$ & $<0,01$ \\
\hline Fiebre** & 2 & 7 & $2,33(1,23-4,41)$ & $<0,05$ \\
\hline \multicolumn{5}{|l|}{ Análisis multivariado } \\
\hline SOFA $>3$ & & & $24,9(2,3-267,7)$ & $<0,01$ \\
\hline Corticosteroides sistémicos con anfotericina B & & & $7,56(1,20-47,5)$ & $<0,05$ \\
\hline
\end{tabular}


Reacciones adversas durante la infusión: En este trabajo, las reacciones adversas adscritas a la infusión de anfotericina B tales como la fiebre, vómitos, calofríos o la flebitis fueron frecuentes, siendo la primera de ellas, la forma predominante $(25 \%)$ y alcanzando en conjunto a $40 \%$ de los tratamientos. Un estudio previo sobre reacciones adversas asociadas a infusiones de anfotericina $\mathrm{B}$ deoxicolato administradas a distintas velocidades (infusión lenta de dos horas o rápida de 45 minutos), demostró que estas manifestaciones son muy frecuentes, alcanzando valores de $70 \%$ para la fiebre y de $80 \%$ para calofríos ${ }^{17}$. Las cifras superiores reportadas por estos autores podrían ser explicadas porque las velocidades de infusión utilizadas (45 minutos y dos horas) corresponden en realidad a infusiones rápidas. En otro estudio randomizado no se encontraron diferencias significativas para estos fenómenos al comparar infusiones de una y cuatro horas ${ }^{18}$. Actualmente, se propone aplicar infusiones con velocidades sobre las cuatro horas para disminuir la gravedad y frecuencia de estas reacciones adversas ${ }^{14,19}$. Nuestra tasa de reacciones adversas fue similar a la encontrada por Hernández y cols, en la rama de pacientes que recibió anfotericina $\mathrm{B}$ deoxicolato $(47 \%)$ y a los tres casos afectados por ellas en 10 tratamientos descritos por Espinoza y cols ${ }^{11,12}$. Desafortunadamente, y probablemente por el bajo tamaño de muestra en este trabajo, no fue posible demostrar que aquellos pacientes que recibieron infusión lenta en 24 horas tenían menos efectos adversos que los que recibieron infusiones de cuatro a seis horas, tal como ha sido previamente reportado ${ }^{19}$. De todas maneras, es notoria la alta frecuencia con que estos nuevos protocolos de administración son utilizados en la práctica cotidiana. La frecuencia de vómitos y calofríos fue baja y sólo dos pacientes desarrollaron flebitis en el sitio de infusión periférico. Ello se explica porque casi todos los pacientes recibieron anfotericina $B$ por un catéter central.

Que las características del paciente (edad mayor) y no la forma de administración aparezcan ligadas al desarrollo de reacciones adversas, indica que las manifestaciones observadas en esta población responden probablemente más al proceso mórbido del paciente que a estos protocolos. Este hallazgo no permite asignar en forma inequívoca estos eventos como reacciones adversas del medicamento.

Nefrotoxicidad. La frecuencia reportada de nefrotoxicidad por anfotericina B deoxicolato ha sido muy variada, comunicándose una disminución significativa de la función renal hasta en $80 \%$ de los pacientes tratados con este compuesto $^{20}$, y en forma proporcional a la dosis, siendo casi inevitable en los que reciben una dosis acumulada mayor a 5 gramos $^{21}$. Sin embargo, en la actualidad se indican dosis totales mucho más bajas y por lo tanto, el riesgo de nefrotoxicidad disminuye ${ }^{21}$.

En nuestro grupo, sólo tres de 39 tratamientos $(9,4 \%)$ se asociaron al desarrollo de nefrotoxicidad de acuerdo a los criterios definidos y todos ellos en pacientes con función renal previa normal. En la década de los 80 se describió nefrotoxicidad en $20 \%$ de una pequeña serie de 10 pacientes en nuestro país ${ }^{12}$. La ausencia de nefrotoxicidad en el grupo con disfunción previa en nuestro trabajo se explica por el uso de criterios más exigentes para definirla. Cabe destacar que ninguno de estos pacientes requirió iniciar diálisis y que los aumentos de creatininemia, aunque significativos, fueron discretos. Este hecho desmitifica el perfil renal adverso de esta molécula, tanto por el bajo porcentaje de personas que sufren nefrotoxicidad y por lo discreto de la alteración en la función renal, manteniendo la vigencia de anfotericina B deoxicolato como alternativa terapéutica en una serie de escenarios clínicos. En rigor, las reacciones adversas asociadas a la infusión fueron más frecuentes que la nefrotoxicidad y en ellas, por lo tanto, reside su mayor desventaja. Además, la nefrotoxicidad es un evento que en parte se puede prevenir y al menos monitorizar. Estas conclusiones son importantes en el escenario actual donde la promoción de otros compuestos antifúngicos descansa para algunas condiciones, más en su perfil de seguridad que una mayor eficacia clínica y a expensas de un elevado costo.

Varios factores de riesgo han sido asociados al desarrollo de nefrotoxicidad por anfotericina B: edad mayor, dosis diaria o dosis acumulada elevada, duración prolongada del tratamiento, uso de otros nefrotóxicos concomitantes, shock al inicio del tratamiento, no uso de corticosteroides y una velocidad de infusión corta ${ }^{19,20,22}$. Sin embargo, en nuestro trabajo, no pudimos correlacionar la aparición de nefrotoxicidad con estas variables clínicas. La velocidad de infusión ha demostrado ser un importante determinante del desarrollo de toxicidad renal, tal como lo demostrara Ericsson y cols, a través de un estudio prospectivo randomizado comparando infusiones de cuatro versus 24 horas. En tal estudio se observó una reducción significativa de nefrotoxicidad en pacientes que recibieron anfotericina $\mathrm{B}$ en infusión continua (24 horas) versus aquellos con infusión rápida (cuatro horas) ${ }^{19}$. La baja cifra observada en nuestro trabajo indica que este compuesto mantiene su sitial terapéutico en ciertas infecciones o sospecha de ellas, a pesar de los cuestionamientos a su toxicidad renal.

Hipokalemia. Esta complicación se presentó en ocho tratamientos $(21,6 \%)$, demostrando una tasa relativamente baja para esta reacción, en contraste con la experiencia de Cruz y cols, que utilizando el mismo criterio, evidenció esta complicación en todos sus pacientes ${ }^{14}$. La frecuencia de hipokalemia en el trabajo nacional de Hernández y cols, alcanzó a $52 \%$ y $90 \%$ en el de Espinoza y cols ${ }^{11,12}$. Las diferencias pueden explicarse por el tipo de paciente (sólo pacientes críticos en el trabajo de Hernández), las definiciones utilizadas, el momento escogido para evaluarlo o las intervenciones correctivas ${ }^{11}$. 
Indicaciones de tratamiento. Una amplia diversidad de condiciones estuvo asociada al uso de anfotericina B deoxicolato en nuestra institución, siendo utilizada exclusivamente sobre bases empíricas en más de la mitad de los casos. La mayor parte de las prescripciones estuvo relacionada al manejo de una infección confirmada o presunta por hongos filamentosos y en forma secundaria a infecciones por levaduras. El perfil es, en parte, explicado por la existencia de pacientes con neutropenia febril persistente con fracaso de un tratamiento antimicrobiano de amplio espectro. A pesar de que el riesgo real de aspergilosis pulmonar invasora no supera al $10 \%$ en este subgrupo, se aplica anfotericina B en forma precoz para evitar las consecuencias devastadoras de esta infección fúngica. Anfotericina también fue utilizado en infecciones por levaduras que tuvieron limitaciones para el uso de triazoles o en el manejo de cuadros graves de meningitis por $C$. neoformans, indicaciones bien establecidas en la literatura médica ${ }^{23}$. En una minoría, se indicó como medida profiláctica.

Respuesta al tratamiento y condición al egreso. En el subgrupo con terapia dirigida por información microbiológica, se pudo apreciar que predominó una respuesta no favorable, tanto para infecciones por hongos filamentosos como por levaduras, evidenciando las serias dificultades que tiene la terapia antifúngica para controlar estas infecciones. Estas limitaciones nacen del tipo de pacientes afectados (enfermedad primaria y co-morbilidad), la oportunidad diagnóstica (y por lo tanto, en el inicio del tratamiento), la gravedad y el sitio afectado. A pesar de su espectro de acción privilegiado, anfotericina B tiene una eficacia clínica sub-óptima para el manejo de infecciones fúngicas invasoras, especialmente aspergilosis, donde su eficacia no supera el $40-50 \%$ y esta eficacia no parece aumentar con un incremento de las dosis o con el uso de las formas lipídicas ${ }^{9,24-26}$. En un estudio realizado a pacientes con sospecha o diagnóstico confirmado de aspergilosis se documentó una mortalidad de $60 \%$ asociada a factores tales como el uso de otros agentes nefrotóxicos o inmunosupresore ${ }^{27}$. De la misma manera, en nuestro trabajo, luego de un análisis multivariado, se lograron encontrar factores asociados a una evolución letal que correspondieron a un puntaje SOFA $>3$ y al uso concomitante de corticosteroides sistémicos en la terapia. Estos hallazgos no son discordantes con la experiencia clínica cotidiana y la literatura, incluyendo estudios nacionales de pacientes con $\operatorname{sepsis}^{28-30}$.

Limitaciones del trabajo. Este trabajo tiene varias limitaciones entre las que destacan su naturaleza retrospectiva (que limita la obtención de datos y no utiliza una randomización terapéutica balanceada) y su bajo tamaño muestral (que limita el poder estadístico de los hallazgos). No obstante, da cuenta de la realidad de las condiciones asociadas al uso de anfotericina B deoxicolato, sus aciertos y problemas. Por otra parte, no estamos conscientes de que las reacciones adversas observadas en estos pacientes haya alguna vez sido notificada a algún sistema de fármaco-vigilancia activa o pasiva en el país.

\section{Conclusiones}

En la serie analizada se pudo observar que más de la mitad de los tratamientos con anfotericina B fueron realizados sobre bases empíricas, especialmente ante la sospecha de aspergilosis pulmonar invasora en pacientes con neutropenia febril persistente. Anfotericina B es frecuentemente utilizada también para el tratamiento de infecciones por levaduras (Candida o Cryptococcus).

Las reacciones adversas relacionadas a la infusión de anfotericina $\mathrm{B}$ deoxicolato se presentaron en casi la mitad de los pacientes y la nefrotoxicidad tuvo una baja incidencia en nuestro grupo en estudio y de escasa relevancia clínica.

En la mayor de los casos no se observó una respuesta favorable con el uso de anfotericina $\mathrm{B}$, incluso en el grupo con terapia dirigida por información microbiológica o en el subgrupo con infecciones por levaduras.

Se pudo identificar en nuestro trabajo diferentes factores que se asociaron al desarrollo de reacciones adversas durante la infusión y a un desenlace fatal. En esta experiencia, las reacciones adversas no dependieron de los protocolos de administración sino de ciertas propiedades del hospedero.

\section{Resumen}

Anfotericina B deoxicolato se asocia a reacciones adversas durante la infusión y a nefrotoxicidad. Objetivo: Evaluar las indicaciones de anfotericina B deoxicolato en un hospital universitario, las reacciones adversas asociadas, los protocolos de administración y el desenlace de los pacientes tratados. Pacientes y Métodos: Se efectuó un estudio retrospectivo con el total de tratamientos efectuados durante el año 2007 en el Hospital Clínico de la Universidad de Chile, identificando 39 tratamientos en 33 pacientes. Se analizaron las indicaciones, dosis, protocolos de administración, efectos adversos relacionados a la infusión (fiebre, calofríos, vómitos o flebitis), nefrotoxicidad, hipokalemia y además la evolución de los pacientes. Resultados: La duración promedio del tratamiento fue de 12 días (2-39) con una dosis acumulada promedio de $600 \mathrm{mg}$ totales (100-1.950 mg). Un 63,2\% de los tratados recibió infusiones de 24 horas y 35,9\%, infusiones de 4 a 6 horas. Además, $36,8 \%$ fue sometido a precargas salinas. 
Un $40 \%$ de los tratamientos se acompañó de reacciones adversas asociadas a la infusión, predominando la fiebre $(25 \%)$. Sin embargo, la nefrotoxicidad fue de baja magnitud $(9,4 \%)$, sólo presente en pacientes sin falla renal previa y en ningún caso determinó el inicio de diálisis. La hipokalemia se presentó en ocho tratamientos $(21,6 \%)$. Más de la mitad de las indicaciones fueron empíricas $(59 \%)$, ya fuese para el tratamiento presunto de hongos filamentosos (aspergilosis o mucormicosis) o levaduras (candidiasis sistémica). En el subgrupo con datos microbiológicos, las principales indicaciones fueron aspergilosis invasora ( $15,4 \%$ de los 39 tratamientos), candidiasis sistémica $(12,8 \%)$ o criptococosis meníngea $(10,3 \%)$.
Un $41 \%$ de los pacientes tuvo una respuesta favorable a los tratamientos y sólo 48,5\% sobrevivió. En un análisis multivariado, sólo la edad $>60$ años permaneció como un factor independiente ligado al desarrollo de reacciones adversas asociadas a la infusión y no los protocolos de administración. Este mismo enfoque permitió asignar un puntaje SOFA $>3$ y el uso concomitante de corticosteroides sistémicos como factores independientes para un desenlace fatal. Conclusión: Las reacciones adversas asociadas a la infusión de anfotericina $\mathrm{B}$ deoxicolato son fenómenos frecuentes pero la nefrotoxicidad es ocasional y de baja magnitud. Anfotericina B se usa predominantemente sobre bases empíricas.

\section{Referencias}

1.- Santolaya M E, Rabagliatti R, Bidart T, Payá E, Guzmán A M, Morales R, et al. Consenso manejo racional del paciente con cáncer, neutropenia y fiebre. Rev Chil Infect 2005; 22 (Supl2): S79-113.

2.- Silva V, Díaz M C, Febré N, Chilean Invasive Fungal Infection Group. Invasive fungal infections in Chile: A multicenter study of fungal prevalence and susceptibility during 1-year period. Med Mycol 2004; 42: 333-9.

3.- Fica A. Infecciones en el paciente con trasplante de órganos sólidos. Rev Hosp Clín Univ Chile 2007; 18: 346-62.

4.- Mazuski J E, Sawyer R G, Nathens A B, DiPiro J T, Schein M, Kudsk K A, et al. The surgical infection society guidelines on antimicrobial therapy for intra-abdominal infections: Evidence for the recommendations. Surg Infect 2002; 3: 175-233.

5.- Gallis H A, Drew R H, Packard W W Amphotericin B: 30 years of clinical experience. Rev Infect Dis 1990; 12: 308-29.

6.- Graybill J, Craven P. Antifungal agents used in systemic mycoses. Activity and therapeutic use. Drug 1983; 25: 41-62.

7.- Rex J H, Bennett J E, Sugar A M, Pappas P G, van der Horst C M, Edwards J E, et al. A randomized trial comparing fluconazole with amphotericin B for the treatment of candidemia in patients without neutropenia. Candidemia Study Group and The National Institute. N Engl J Med 1994; 331: 1325-30.

8.- Herbrecht R, Denning D W, Paterson T F, Bennet J E, Greene R E, Oestmann J E, et al. Voriconazole versus amphotericin B for primary therapy for invasive aspergillosis. N Engl J Med 2002; 347: 408-15.

9.- Fica A, Tratamientos de infecciones fúngicas sistémicas: Anfotericina $\mathrm{B}$, aspectos fármaco económicos y decisiones terapéuticas. Rev Chil Infect 2004; 21: 317-26.

10.- Cruz Ch R. Comentarios sobre la eficacia y nefrotoxicidad de anfotericina B convencional e intralipídica en adultos con infección fúngica sistémica y compromiso inmune. Boletín Micológico 2004; 19: 31-5.

11.- Hernández G, Altermatt F C, Bernucci F P, Acuña D C, Apablaza F E. Uso de anfotericina B en mezcla lipídica: ipreviene la toxicidad de la droga en pacientes críticos? Rev Méd Chile 2000; 128: 1101-7.

12.- Espinoza R, Acuña G. Uso clínico de anfotericina B. Rev Chil Infect 1986; 3: 119-22.

13.- Vincent J L, Moreno R, Takala J, Willatts S, De Mendonça A, Bruining $\mathrm{H}$, et al. The SOFA (sepsis-related organ failure assessment) score to describe organ dysfuntion/failure. Intensive Care Med 1996; 22 : 707-10.

14.- Cruz J, Peacock J. Rapid intravenous infusion of amphotericin B: Pilot study. Am J Med 1992; 93: 123-30.

15.- Michalopoulos A S, Tsiodras S, Rellos K, Mentzelopoulos S, Falagas M E. Colistin treatment in patients with ICU-acquired infections caused by multiresistant Gram-negative bacteria: the renaissance of an old antibiotic. Clin Microbiol Infect 2005; 11: 115-21.

16.- Ascioglu S, Rex J H, de Pauw B, Bennett J E, Bille J, Crokaert F, et al. Defining opportunistic invasive fungal infections in immunocompromised patients with cancer and hematopoietic ítem cell transplants: An internacional consensus. Clin Infect Dis 2002; 34: 7-14.

17.- Cleary J D, Weisdorf D, Fletcher C. V. Effect of infusion rate on amphotericin B - associated febrile reactions. Drug Intell Clin Pharm 1988; 22: 769-72.

18.- Oldfield III E C, Garst P D, Hostettler C, White M, Samuelson D. Randomized, double- blind trial of 1 - versus 4-hour amphotericin B infusion durations. Antimicrob Agents Chemother 1990; 34: 1402-6.

19.- Ericsson U, Seifert B, Schaffner A. Comparison of effects of amphotericin B deoxycholate infused over 4 hours or 24 hours: randomized controlled trial. Br Med J 2001; 322: 1-6.

20.- Luber A, Maa L, Lam M, Guglielmo J B. Risk factors for amphotericin B- induced nephrotox- icity. Antimicrob Agents Chemother 1999; 43 : 267-71.

21.- Bates D, Su L. Correlates of acute renal failure in patients receiving parenteral amphotericin B. Kidney Int 2001; 60: 1452-9.

22.- Harbarth S, Pestotnik M, Lloyd J, Burke J, Samore M. The epidemiology of nephrotoxicity associated with conventional amphotericin B therapy. Am J Med 2001; 11: 528-34.

23.- Saag M S, Powderly W G, Cloud G A, Robinson P, Griego M H, Sharkey P K, et al. Comparison of amphotericin B with fluconazole in the treatment of acute AIDS-associated cryptococcal meningitis. N Engl J Med 1992; 326: 83-9.

24.- Thipine M, Letscher-Bru V, Herbrecht R. Amphotericin B and its new formulations: pharmacologic characteristics, clinical efficacy, and tolerability. Transpl Infect Dis 1999; 1: 273-83.

25.- Gotzsche P C, Johansen H K. Meta-analysis of prophylactic or empirical antifungal treatment versus placebo or no treatment in patients with cancer complicated by neutropenia. Br Med J 1997; 314: 1238-44.

26.- Ellis M. Amphotericin B preparations: a maximum tolerated dose in severe invasive fungal infections? Transpl Infect Dis 2000; 2: 51-61.

27.- Wingard J, Kubilis P, Lee L, Yee G, White M, et al. Clinical significance of nephrotoxicity in patients treated with amphotericin B suspected or proven aspergillosis. Clin Infect Dis 1999; 29: 1402- 7 .

28.- Dougnac A, Mercado M, Cornejo R, Cariaga M, Hernández G, Andresen M, et al. Prevalencia de sepsis grave en las unidades de cuidados intensivos. Primer estudio nacional multicéntrico. Rev Méd Chile 2007, 135: 620-30.

29.- Minne L, Abu-Hanna A, de Jonge E. Evaluation of SOFA-based models for predicting mortality in the ICU: a systematic review. Crit Care 2008; 12: R161(doi 10.1186/cc7160).

30.- Janssen N M, Karnad D R, Guntupalli K K. Rheumatologic diseases in the intensive care unit: epidemiology, clinical approach, management, and outcome. Crit Care Clin 2002; 18: 729-48. 